\title{
Peer mentoring: An effective strategy for integrating HIV and SRH
} services

Integra Initiative

Follow this and additional works at: https://knowledgecommons.popcouncil.org/departments_sbsr-rh

Part of the Demography, Population, and Ecology Commons, Family, Life Course, and Society Commons, International Public Health Commons, and the Women's Health Commons How does access to this work benefit you? Let us know!

\section{Recommended Citation}

"Peer mentoring: An effective strategy for integrating HIV and SRH services," Steps to Integration, Issue 2. Washington, DC: Integra Initiative, 2015. 


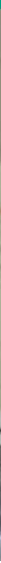

This Steps to Integration series provides a guide on how to integrate HIV and sexual and reproductive health (SRH) services based on findings from the Integra Initiative.

The Integra Initiative is a research project on the benefits and costs of a range of models for delivering integrated HIV and SRH services in high and medium HIV prevalence settings, to reduce HIV infection (and associated stigma) and unintended pregnancies. It was managed by the International Planned Parenthood Federation (IPPF) in partnership with the London School of Hygiene \& Tropical Medicine (LSHTM) and the Population Council.

Findings from the project show that integrating HIV and SRH services has the potential to:

\section{$\checkmark$ Increase uptake of health} services

$\checkmark$ Increase range of services available

$\checkmark$ Improve quality of services and efficient use of resources

$\checkmark$ Enable health systems to respond to client needs and improve overall client satisfaction

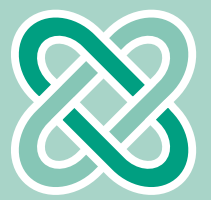

www.integrainitiative.org
Peer mentoring: an effective strategy for integrating HIV and SRH services

\section{Introduction}

Integrating HIV and sexual and reproductive health (SRH) services is essential for effective health programming. One Integra Initiative component explored how peer mentoring can be an effective, feasible and sustainable tool to support in-service capacity-building.

However, integration remains limited because knowledge and skills gaps among frontline providers constrain their ability to provide essential services. Further, traditional capacity-building approaches, for example offsite training workshops, are costly, are not conducive to knowledge-sharing among colleagues and interrupt service provision.

Mentoring is an innovative approach to improving provider skills without compromising service delivery by harnessing the potential of existing providers. Mentorship occurs when a more skilled or experienced person (mentor) is paired with a less skilled person (mentee), with the agreed-upon goal of developing the latter's abilities.

In Kenya, as part of the Integra Initiative, a peer mentorship approach was designed and tested to improve service providers' skills, knowledge and capacity to provide high quality integrated HIV and SRH services. These activities took place between August 2009 and June 2010. A central component was to

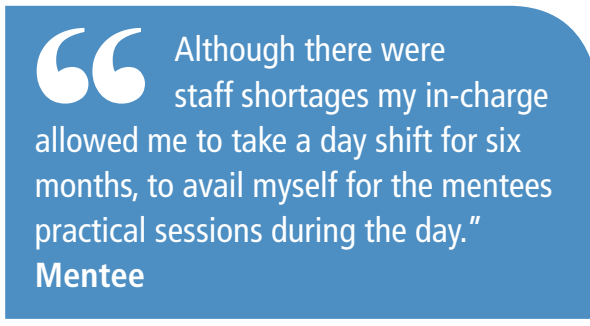
understand providers' opinions on mentoring, so as to build a strong and feasible mentorship model that would be practical as well as sustainable. 


\section{The mentoring process}

Jointly with the Kenya Ministry of Health $(\mathrm{MOH})$, the Integra Initiative developed, implemented and assessed a mentorship programme (see Figure 1 below).

\section{Figure 1: Mentorship programme development and implementation}

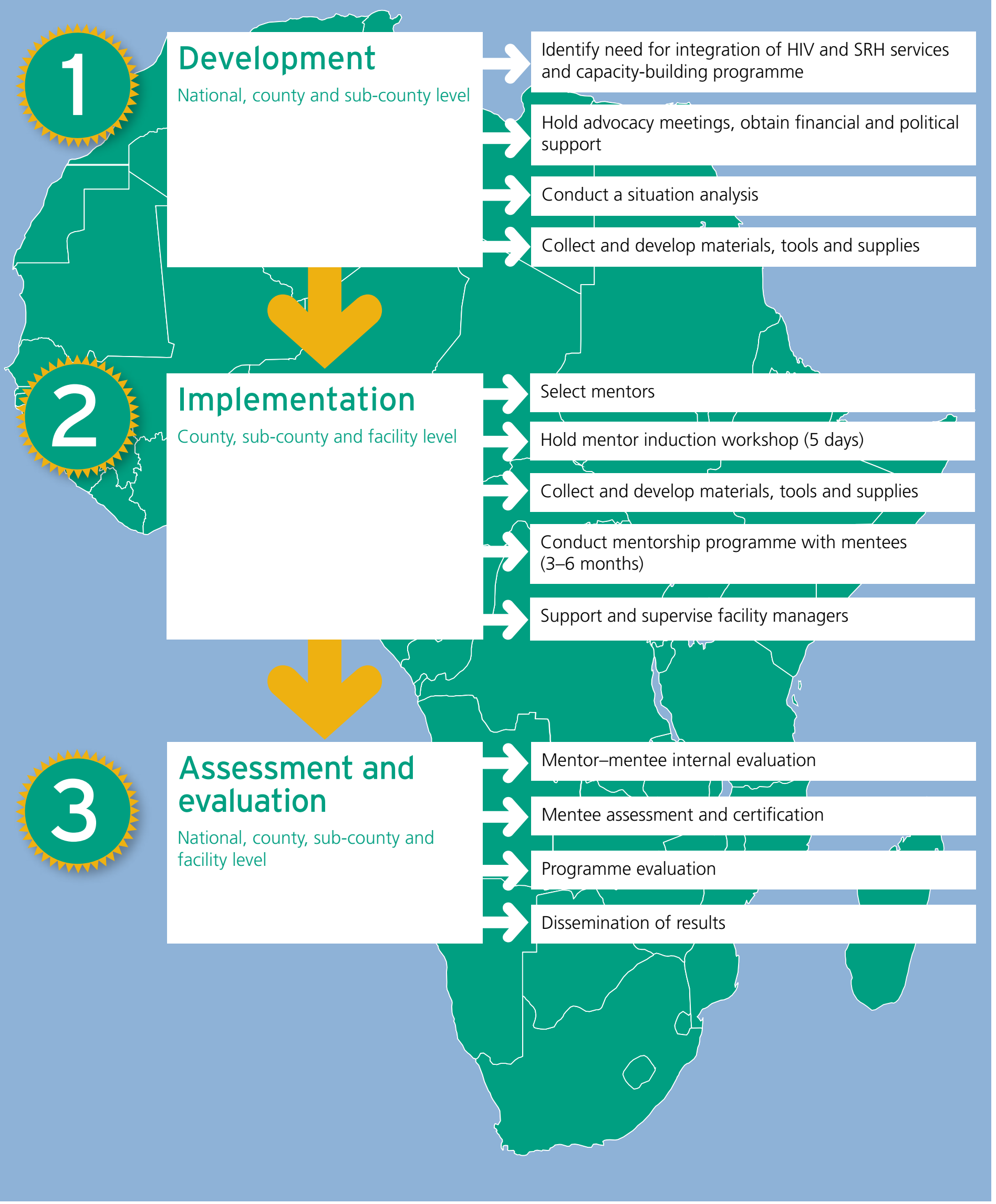




\section{Mentorship tools}

\section{RH/HIV Integrated Service Provision in Kenya}

This toolkit was developed by the project team and consists of a trainer's manual, participant's manual, mentee initial assessment pre-test, mentees clinical protocol, mentor logbook, monthly summary sheet, integrated RH/HIV clinical assessment tool and mentorship guidelines.

The Guide for Mentors provides an overview of the programme and serves as the primary document for the mentor to use during the Mentor's Induction Workshop and Mentorship Programme. The guide is divided into two sections. Section 1 outlines content for mentoring exercises and lessons in the Mentor's Induction Workshop. Section 2 walks the mentors through classroom and practical lessons included in both the Mentor's Induction Workshop and Mentorship Programme. Tools and references, including relevant national RH/HIV guidelines, are outlined in each training session.
The Guidelines for Mentees serve as the primary resource for mentees during the Mentorship Programme. Course content for each lesson and exercise is clearly written out in each training session as well as references to guidelines for relevant RH/HIV clinical skills. Mentees should refer to this document during the Mentorship Programme and may find it useful to reference even after the programme is complete.

The Integrated RHIHIV Clinical Assessment Tool is an initial assessment form that is completed by every new mentee before starting the Mentorship Programme and submitted to the mentor. This form acts as a baseline and is compared to subsequent assessments.

\section{Balanced Counseling Strategy Plus (BCS+)}

Another integration tool used in the training is the Balanced Counseling Strategy Plus (BCS+), an interactive, client-friendly approach for improving counseling on family planning and prevention, detection and treatment of sexually transmitted infections including HIV.

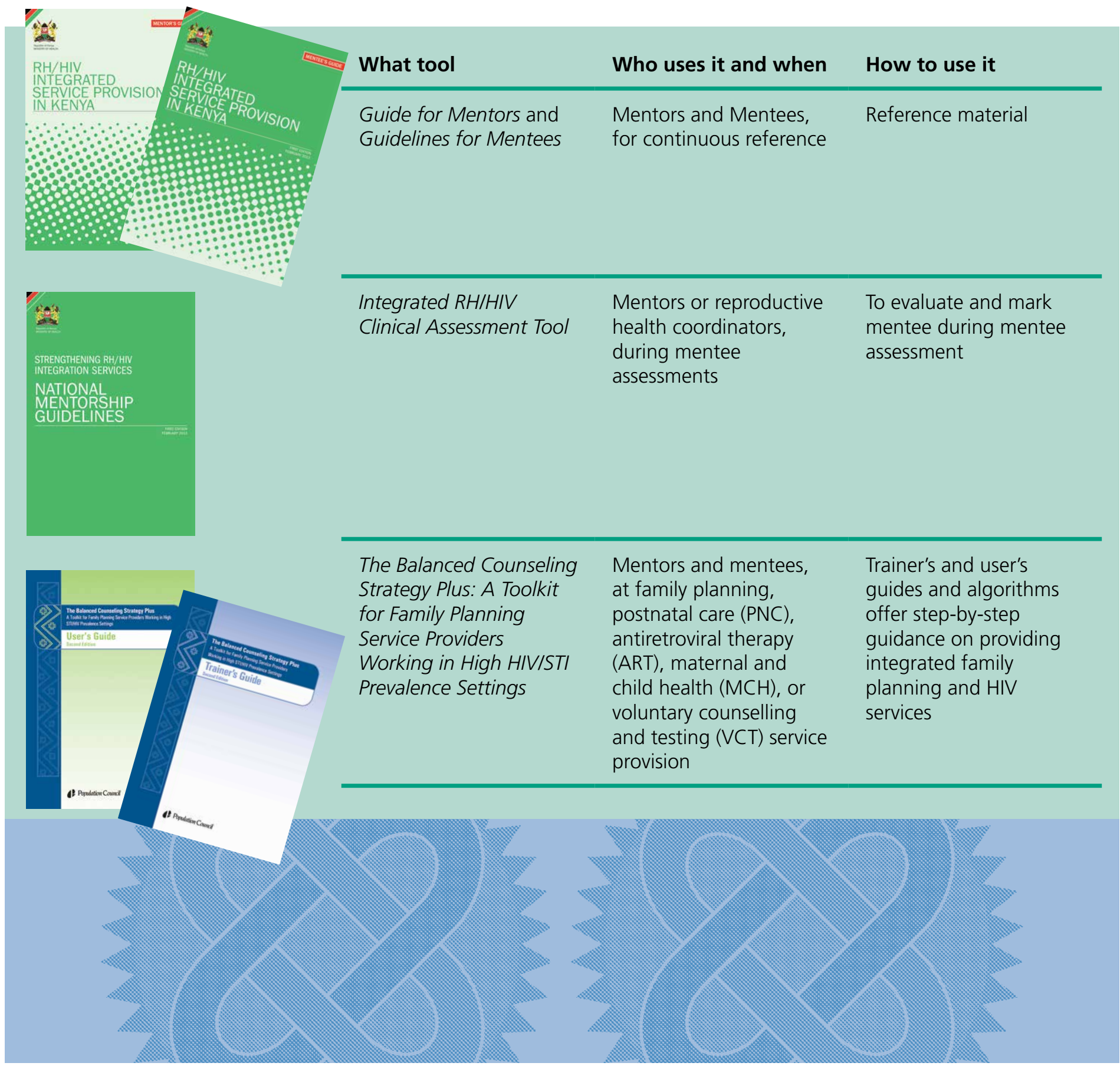


